\title{
Addressing Detector Non-Uniformity in Scanning Transmission Electron Microscopy
}

\author{
J. M. LeBeau ${ }^{1}$ and S. D. Findlay ${ }^{2}$ \\ 1. North Carolina State University, Department of Materials Science and Engineering, Raleigh, NC \\ 27695, USA
}

2. School of Physics, Monash University, Victoria 3800, Australia

Recent years have seen a rise in the number of atomic-resolution scanning transmission electron microscopy (STEM) investigations involving quantitative comparisons between experimentally recorded and simulated images [1-5]. In one approach, quantitative comparisons between simulation and experiment are based on relative contrast measures such as peak height ratios and profile shape. Alternatively, direct comparisons between experiment and simulation can be achieved by quantitative analysis of the absolute signal. Absolute quantification requires, however, that several microscope parameters be appropriately characterized.

In this talk, we will show that even if the detector response is assumed to be uniform, an uncertainty in the detector inner angle of a few mrad can produce a $10 \%$ error in the prediction or interpretation of the peak intensity [3]. Furthermore, we will explore the impact of a typical non-uniform detector response as shown in Fig 1. We will show that for high angle dark field imaging the intensity can be correctly normalized against simulations that assume a uniform detector response corrected by a proper normalization factor. For a typical detector that sits in the lower angle dark field and/or bright field regions, the directional nature of the non-uniformity produces variations in contrast of the order of $510 \%$ relative to a uniform detector. Finally, we will also demonstrate that for annular bright-field imaging, non-uniformity of the detector response can lead to visible qualitative variations in the form of the image, see Fig. 1(f).

As implicit in the simulations presented in this talk, accurate theoretical modeling is always possible for a sufficiently-well-characterized detector by including the measured, non-uniform detector response into the simulations. The similarity between the detector responses that have been reported in the literature (Rosenauer et al. [4], Katz-Boon et al. [5], LeBeau and Stemmer [1-2]) indicates that non-uniformity is common (a result of design geometry) and indicates the need to conduct careful experiments. We will conclude with guidelines for quantifying the absolute intensity to within the $10 \%$ level, especially for annular detectors placed at lower scattering angles.

\section{References:}

[1] J. M. LeBeau, et al. Phys. Rev. Lett. 100 (2008), 206101.

[2] J. M. LeBeau, et al. Nano Letters 10 (2010) 4405-4408.

[3] S. D. Findlay and J. M. LeBeau. Ultramicroscopy 124 (2013), 52-60.

[4] A. Rosenauer, et al. Ultramicroscopy 109 (2009), 1171-1182.

[5] H. Katz-Boon, et al. Ultramicroscopy 124 (2013), 61-70.

[6] S.D.F. acknowledges support by the Australian Research Council and J.M.L. acknowledges support from the state of North Carolina. This work made use of the MRL Central Facilities, supported by the MRSEC Program of the NSF under Award No. DMR 1121053. 

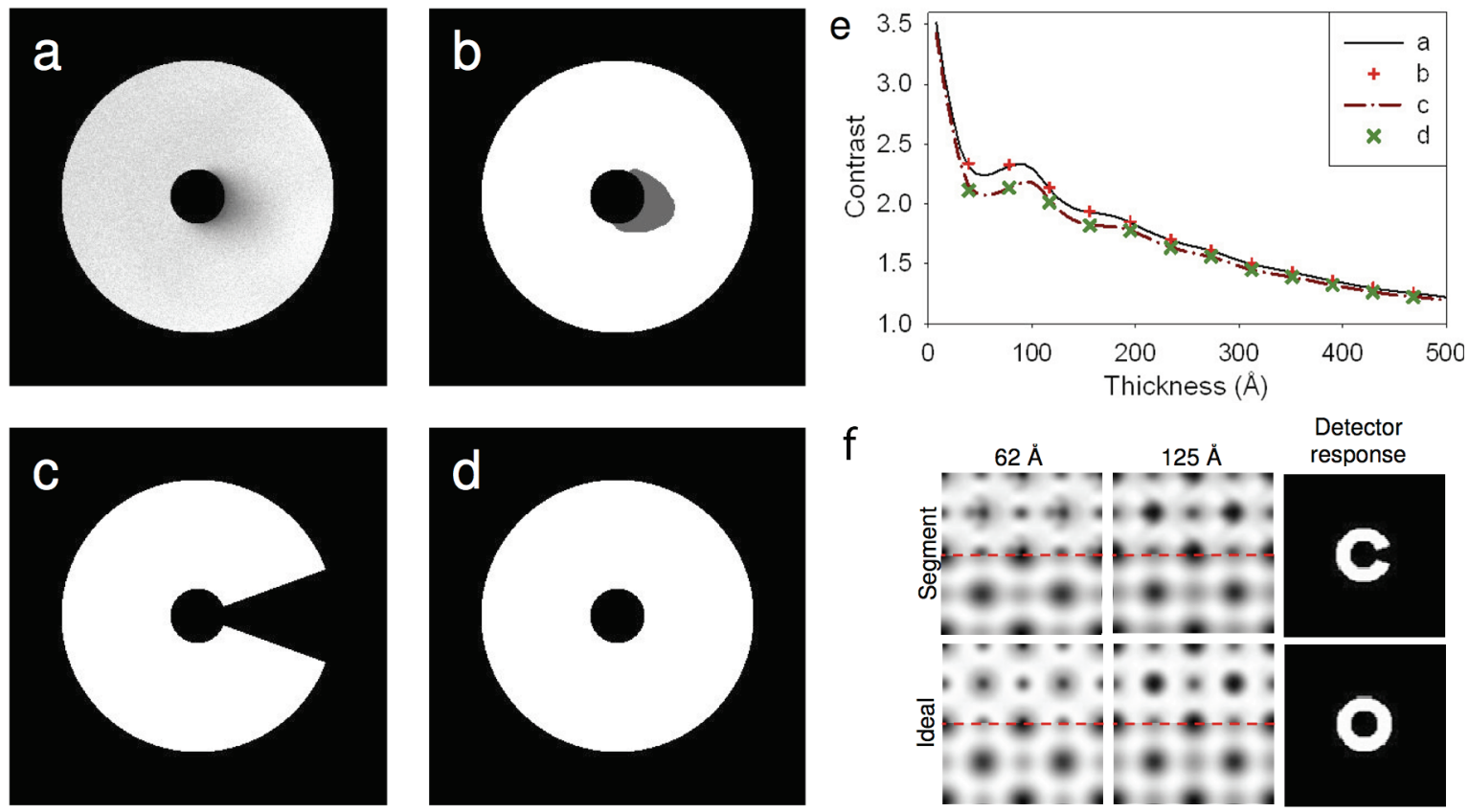

\section{f}

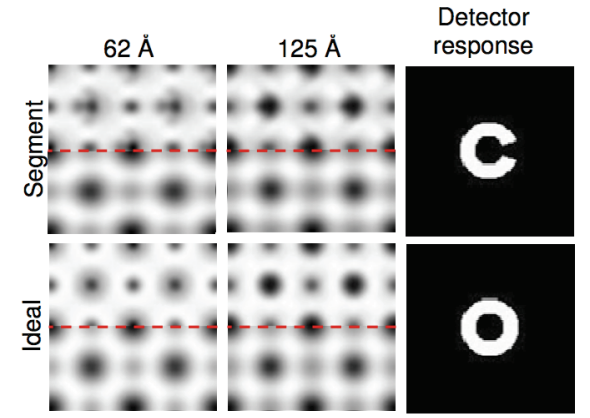

Figure 1. Detector response functions: (a) experimentally measured, (b) two-level approximation to that experimentally measured, (c) uniform with missing a segment, and (d) uniform. (e) Contrast as a function of thickness for a $\mathrm{SrTiO}_{3}$ crystal (aberration-corrected) with the four detector response functions with a collection range of 25-125 mrad. (f) Anisotropic image distortion due to the influence of a segmented detector.

Aberration-corrected probe

Uncorrected probe
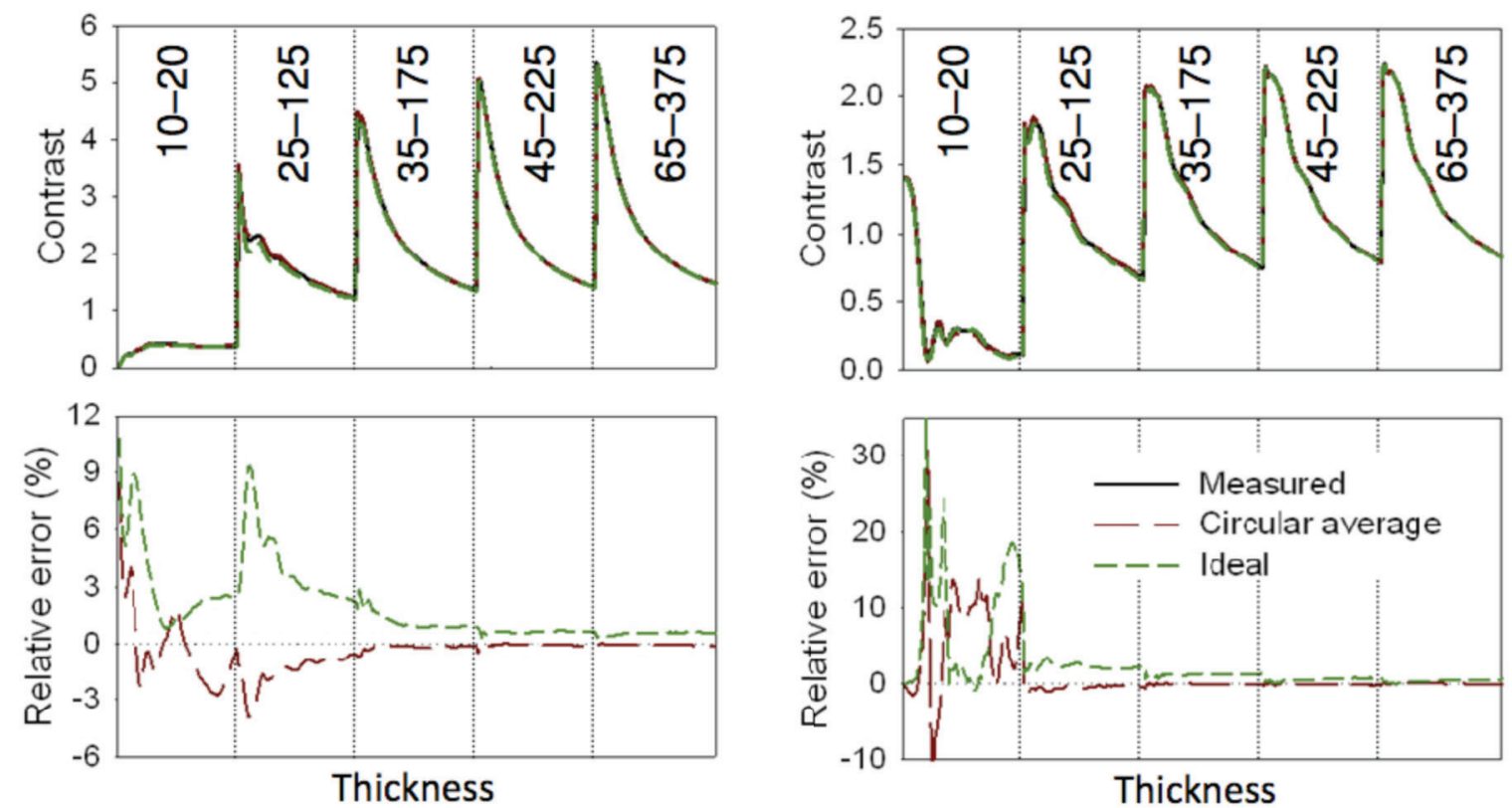

Thickness

Figure 2. A comparison of the real detector response, the circularly averaged detector response, and the ideal, uniform detector response for both an aberration-corrected and an uncorrected probe. Spatial incoherence is included. For each camera length (given by the span ranges labeled in the top plots in mrad units), the horizontal axis runs from zero to $500 \AA$. 